\title{
Elaphoide'la boui n. sp., Copépode Harpacticoïde stygobie du Sud-Ouest du Massif Central
}

\author{
R. Rouch1
}

Muts clés : Copepoda, Harpacticoida, espèce nouvelle, faune aquatique souterraine.

La découverte d'une nouvelle espèce d'Elaphoidella, dans une région karstique apparemment bien prospectéc, montre l'intérêt de prendre en compte la zone noyè des karsts si l'on veut réellement connaitre la faune aqualique souterraine des pays calcaires.

Elaphoidella boui n. sp., stygobiont harpacticoïd copepod from the South-West of Massif Central

Kiswords : Copepoda, Halpacticoida, new species, stygobiont aquatic fauna.

A new Elaphoidella species is described from the saturated zone of a karstic area drained by the river Aveyron. This discovery in an apparently well explored country shows the necessity to investigate the saturated zone of karsts in order 10 really know the slygobiont aquat ic fauna of limestone areas.

Comparée aux Pyrénées, la bordure sud-ouest du Massif Central n'a fourni, à ce jour, que fort peu d'Harpacticides stygobies. Chappuis (1953 a) decrit la première espèce souterraine de cette région, Elaphoidella lenuthi meridionalis, d'aprìs des exemplaires provenant de la grotte du Bosc (commune de Saint-Antonin, Tarn et Garonne). Bou (1966), après une prospection intensive des cavités des BasCausses de Limogne et de la bordure nord de la Grésigne (50 grottes visitées), signale la présence de wite espece dans la grotte-aven de Pouzergues, la grotte des Trois Cloches (commune de Penne, Tarn) "I dans l'aven de Combes (commune d'Itzac, Tarn). F. leruthi meridionalis a été également récoltéc (Rouch, 1968) dans l'aven des Arandies (commune (le Penne, Tarn). Toutes ces stations sont situées à proximité des gorges de l'Aveyron. Mais la répartition d'E. lonthi meridionalis dépasse très largement

\footnotetext{
1. Laboratoire souterrain du CNRS, Moulis, 09200 Saint-Girons, France.

Remerciements :

Je remercie le Docteur T. Petkovski pour les précieux renseigne. ments qu'il m'a fournis ainsi que Madame A. Descouens qui a réalisé les figures définitives de cette note.
}

cette région puisqu'elle s'étend sur toule la bordure sud du Massif Central jusque dans la région de Montpellier et que, plus au sud encore, elle va des Pyrénces-Orientales aux Pyrénées-Atlantiques (Rouch 1986). Vers le nord-ouest, elle a été récoltée en Dordogne, dans le Riberacois (Lebreton \& Dussart 1984).

La deuxième espece stygobie connue de la bordure sud-oues 1 du Massif Central est Ceuhlonectes gallicus Chappuis, 1928 a. Tres largement répartie, elle aussi, dans le sud de la France (Rouch 1986), elle n'a été récoltée que dans trois stations du Causse des Gorges de l'Aveyron: la grot te des Trois Cloches, la grotte de la Pyramide el la source du Figuier (commune de Penne, Tarn).

Ces résultats tendraient à prouver la faible richesse spécifique des Harpacticide stygobies de cette région. Bou (1968), après avoir étudié la faune interstitielle des différentes nappes d'alluvions de l'Albigeois, région limitrophe de la précédente. conclut que la faune aquatique hypogée de la vallée du Tarn est beaucoup plus riche que celle des massifs karstiques du sud des Causses de Limogne. Il observe, cependant, que la faune de ces massifs 
karstiques est encore mal connue car les récoltes y ont été limitées à la zone d'infiltration, la zone noyée ne faisant l'objet d'aucune investigation.

Lesı premières données biologiquess sur le karst noyé en relation avec les Gorges de l'Aveyron sont consécutives à l'aménagement, par Bou, de la grotteexsurgence d'Amiel (commune de Penne, Tam) afin d'alimenter en eau la commune de Penne. Parmi les équipements dont bénéficie cette station, figure un dispositif de filtrage destinc à ctudier la dérive des animaux dans un drain du karst noyé de ce secteur.

Deux mois de filtrage, en fin d'étiage (septembre et octobre 1987), lui ont permis de récolter $287 \mathrm{Har}$ pacticides répartis comme suit.

Attheyella crassa, 21 ९ , 1 \%, 1 copépodite (espèce épigée stygophile).

Ceuthonectes gallicus, 3 or o (espece stygobie).

Elaphoidella boui n. sp., 157 \& \&, 32 o o, 72 copépodites (espèce stygobie).

La découverte d'une nouvelle espèce d'Elaphoidella dans le karst noyé du système d'Amiel, confirme la nécessité de prendre en compte cette partie des aquifères karstiques si l'on veut véritablement connaître la faune aquatique hypogée des régions calcaires.

\section{Elaphoidella boui n. sp.}

Lơalité et matériel. - Grotte-exsurgence d'Amiel, commune de Penne, département du Tarn. Filt rages dans la galeric en amont de la résurgence pendant les mois de septembre et uctobre 1987; 157 \& \&, 32 $\sigma \sigma, 72$ copépodites.

Holotype : 1 \&. Paratypes : $3 \uparrow \&, 5 \circ \circ$ (dans la collection de l'auteur).

\section{DESCRIPTION}

Femelle. - Longueur du corps sans les soies furcales : $486 \mu \mathrm{m}$. Bord postérieur des segments du corps lisse dorsalement et ventralement. Segment génital avec, à son bord distal, une ran ée ventrale de spinules interrompue dans sa partie médiane. Antépénultième et avant-dernier segments avec une rangé ininterrompue de spinules au bord distal et ventral. Dernier segment avec, sur la face ventrale, deux séries de trois et deux spinules à la base de chaque branche furcale (fig. 1 b). Bord libre de l'opercule anal orné de très fines spinules (fig. 1 a).
Les branches furcales, divergentes, sont deux fois plus longues que larges; elles sont armées de deux soies apicales, l'interne beaucoup plus développée que l'externe, d'une soie subapicale interne extrê. mement fine, et de deux soies latérales au bord externe, chacune avec une épine à sa base. Une soie, géniculée à sa base, $s$ 'insère au milieu de la face dorsale (fig. $1 \mathrm{c}$ ).

Antennule : composée de huit articles.

Antenne (fig. 3 c): allobasis glabre. Exopodite uniarticulé avec trois addendes. Endopodite uniarticulé avec deux épines et deux séries de spinules au bord interne, cinq épines apicales et une soie au bord externe.

Mandibule (fig. 1 d) : precoxa avec une pars incisiva bidentée, deux lamelles denticulées, une épine et une soie. Palpe biarticulé avec une épine au premier article et cinq addendes au second.

Maxillule (fig. 1 e) : arthrite de la precoxa avec quatre crochets distaux et une soie subapicale. Coxa avec deux soies et une forte épine dont l'extrémité distale est armée de fines spinules. Basis avec trois soies et une forte épine semblable à celle de la coxa. Exopodite et endopodite représentés par une et deux soies.

Maxille (fig. 1 f) : Syncoxa avec deux endites portant chacun trois addendes. Basis avec un fort crochet et deux soies. Endopodite représenté par une soie.

Maxillipède (fig. $1 \mathrm{~g}$ ) : basis glabre. Premier article de l'endopodite avec une rangée de fines spinules; deuxième article avec un fort crochet.

$P_{1}$ (fig. 2a): basis avec deux épines, l'une externe. l'autre interne. Premier article de l'endopodite atteignant l'extrémité distale du deuxième article de l'exopodite ; il est armé d'une épine à l'angle distal interne; deuxième article avec une épine distale interne ; troisieme article avec trois addendes. Exupodite avec les épines usuelles à l'angle distal externe des deux premiers articles; deuxième article avec une épine distale interne ; troisième article avec quatre addendes.

$\mathrm{P}_{2}$ (fig. 2 b) : basis avec une forte épine externe. Endopodite biarticule; ; premier article avec une épine à l'angle distal interne; deuxième article avec deux épines au bord interne, deux épines apicales et une épine subapicale externe.

Troisieme article de l'exopodite avec cinq addendes. 


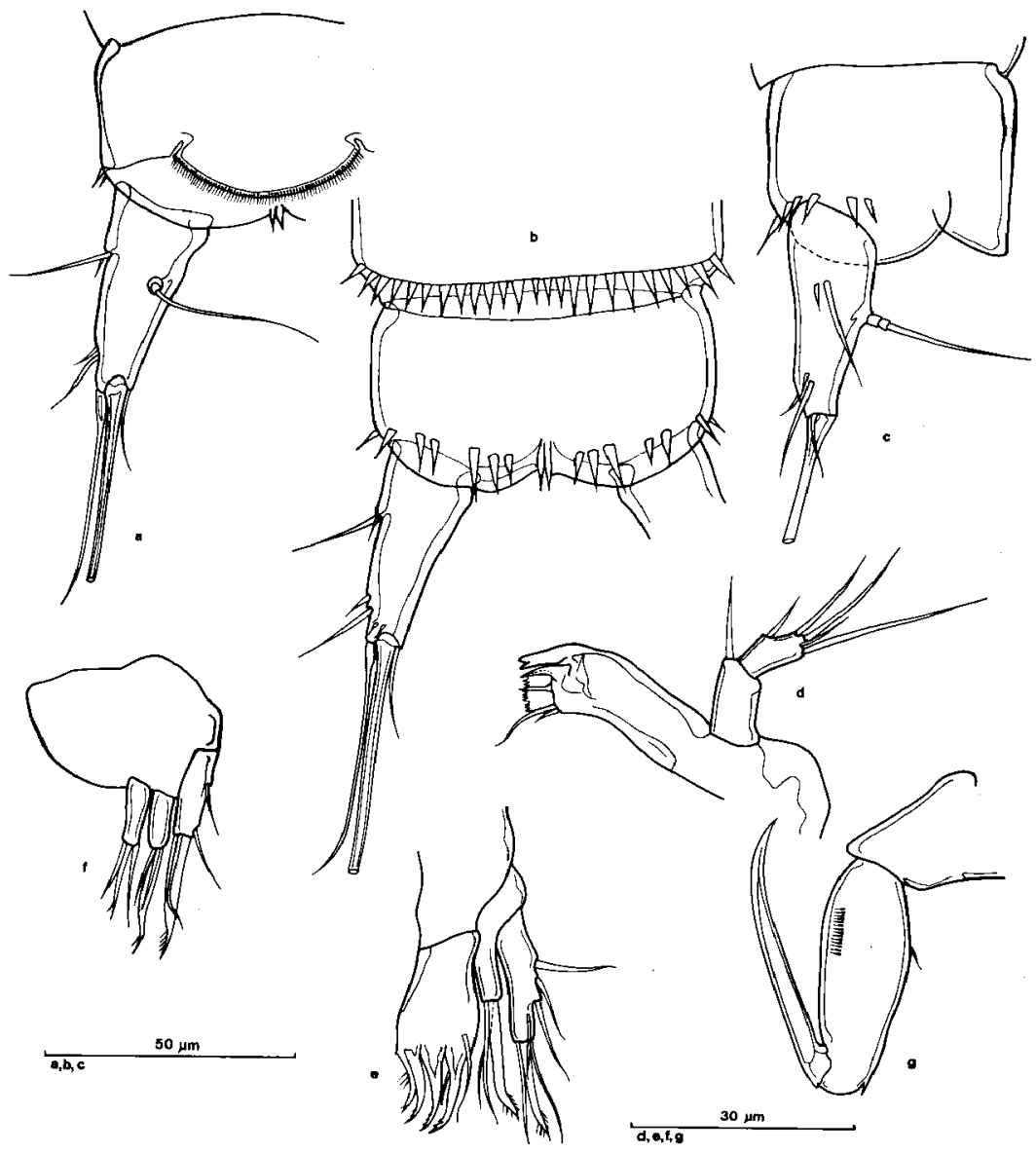

Fig. 1.: Elaphoidella boui n. sp., $Q$ a : dernier segment abdominal et rame furcale, vue dorsale $; \mathrm{b}:$ dernier segment abdominal et rame furcale, vue ventrale ; $c$ dernier segment abdominal et rame furcale, vue latérale : $d:$ mandibule ; $e$ : maxillule; $f$ : maxille; $g$ : maxillipède. 

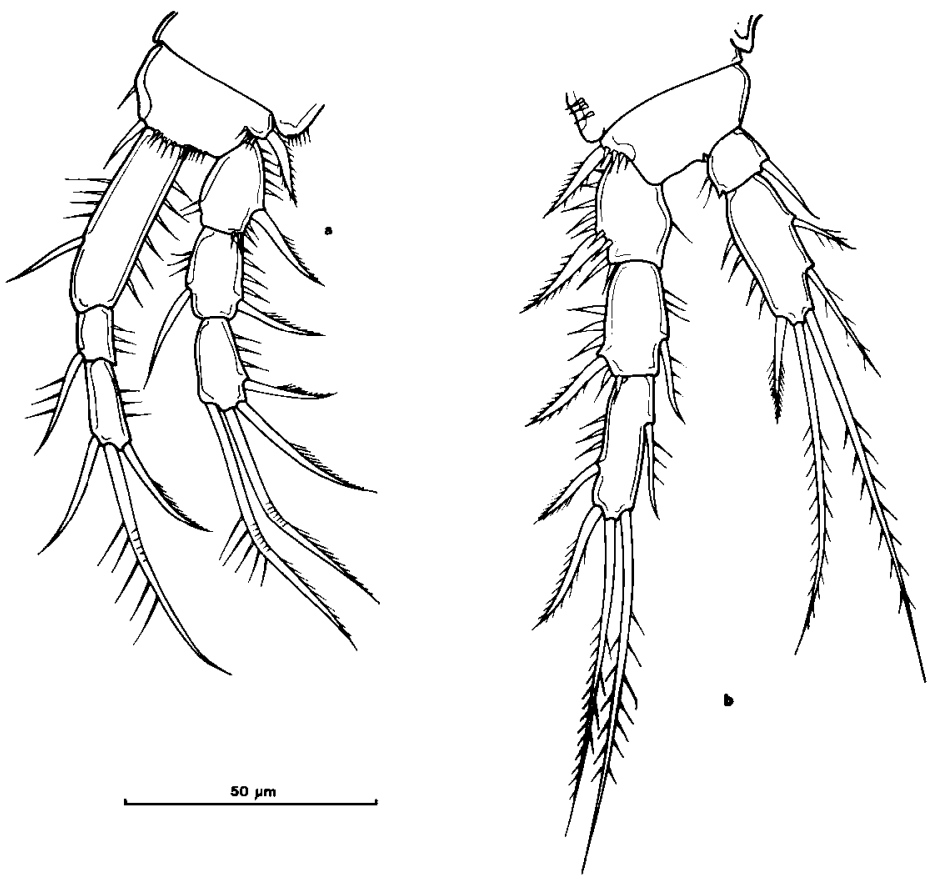

Fig. 2. : Elaphoidella boui n. sp., 9. a : $\mathbf{P}_{1}:$ b : $\mathbf{P}_{2}$.

$P_{3}$ (fig. 3a): basis avec une longue epine externe. Endopodite biarticulé; premier article avec une épine à l'angle distal interne ; deuxième article avec trois épines au bord interne, deux épines apicales et une épine subapicale externe.

Article distal de l'exopodite avec six epines.

$\mathbf{P}_{4}$ (fig. 3 b): basis avec une longue epine externe. Endopodite biarticulé; premier article glabre; deuxieme article avec une épine subapicale interne et deux épines apicales. Article distal de l'exopoditc avec six épines.

La chétotaxie des $P_{1}-P_{4}$ peut être résumée ainsi :
$P_{1} 0.1 .022$
1-1-111
$P_{3}$ 0-1-222
1-321
$\mathrm{P}_{2}$ 0-1-122
$1-221$
$\mathbf{P}_{4}$ 0-1-222
$0-120$

$P_{5}$ (fig. $3 \mathrm{~d}$ ): basévendopodite atteignant la moitié de la longueur de l'sxopodite; il porte quatre épines dont l'externe est la plus longue. Exopodite allongé avec deux épines apicales.

Maile. - Longueur du corps sans les soies furcales: $\mathbf{4 4 2}, \mathrm{m}$. Bord postérieur des segments du corps lisse dorsalement et ventralement. Sixième segment sans ornementation ventrale. Septieme, huitième et neuvieme segments avec une rangée ininterrompue de spinules au bord distal et ventral. Dernier segment et branches furcales comme chez la femelle.

La chétotaxie des $P_{1}$ et $P_{4}$, des exopodiles $P_{2}$ et $P_{3}$ comme chez la femelle. 


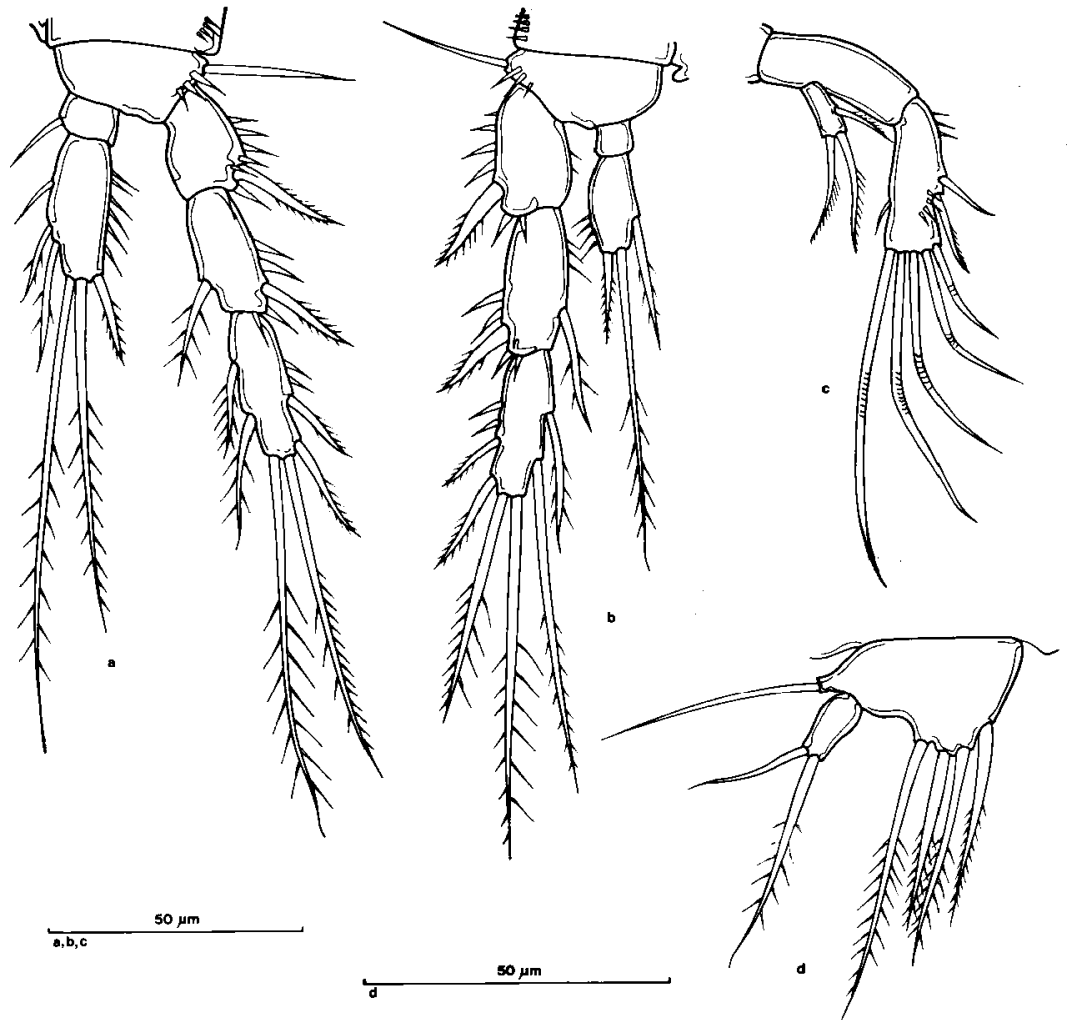

Fig. 3. : Elaphoidella boui n. sp., 8. a: $\mathrm{P}_{3} ; \mathbf{b}: \mathbf{P}_{4} ; \mathbf{c}:$ antenne $; \mathrm{d}: \mathrm{P}_{5}$.

Le dernier article de l'exopodite $\mathrm{P}_{4}$ ne présente aucune épine modifice.

$\mathbf{P}_{2}$ (fig. 4 b) : endopodite biarticulé ; premier article avec une épiné à l'angle distal interne ; deuxième article avec deux epines au bord interne et deux epines apicales.

$P_{3}$ (fig. 4 c) : endopodite triarticulé ; premier article asec une épince au bord interne; deuxicme article avec une longue apophyse lancéolee : troisieme alticle avec deux épines apicales.

$P_{5}$ (fig. 4 a) : baséondopodite de forme Iriangulaire avec une longue épine. Exopodite avec deux épines.

\section{Discussion}

L'exopodite $A_{2}$ uniarticulé, la présence de deux épines seulement au troisieme article des cxopoditcs 

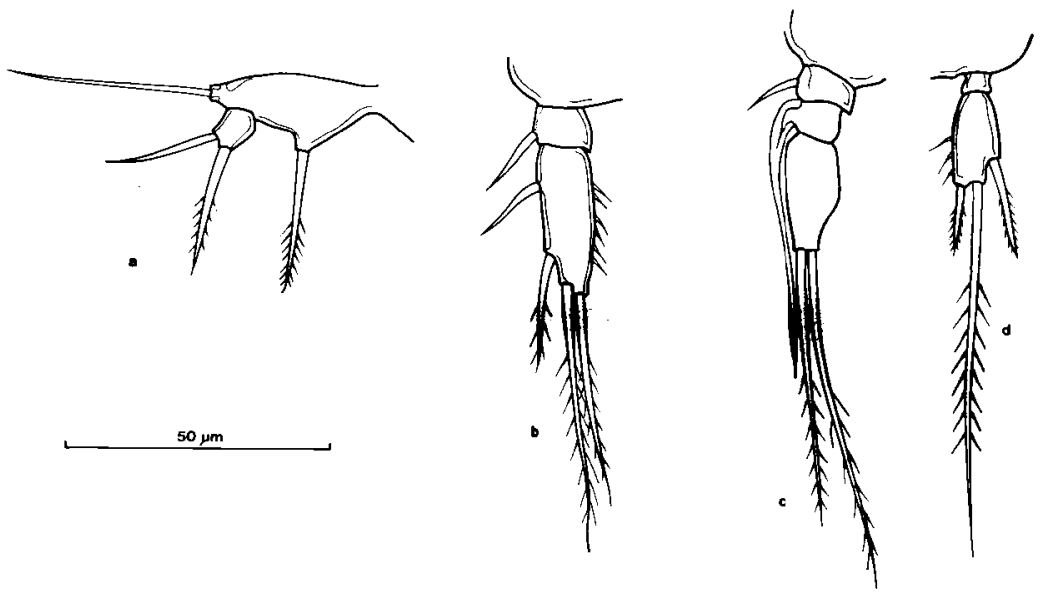

Fig. 4 : Elaphoidella boui n.sp., $\sigma$. - a : $\mathbf{P}_{5} ; \mathbf{b}$ : endopodite $\mathbf{P}_{2} ; \mathbf{c}$ : endopodite $\mathbf{P}_{3} ; \mathbf{d}$ : endopodite $\mathbf{P}_{\mathbf{4}}$.

$\mathbf{P}_{2}, \mathbf{P}_{3}, \mathbf{P}_{4}$, la formule des épines de la $\mathbf{P}_{5} \mathbf{f e m e l l e ~ a v e c}$ dcux épines à l'exopodite et quatre au baséoendopodite sont autant de caractères qui permettent de classer cette espèce dans le genre Elaphoidella.

En revanche, la présence d'une épine bien développée sur le baséoendopodite $P_{5}$ du mâle pourrait faire douter de son appartenance à ce genre. En effet, l'absence d'épine sur le baséoendopodite de la $P_{5}$ du mâle est un des caractères retenus par Chappuis (1929) pour justifier la création du genre Elaphoidella. Lang (1948, p. 1123) atténue la proposition de Chappuis en indiquant que le basis de la $P_{5}$ du mâle est * immer ohne wohlentwickelte Borsten ". En effet, parmi les espèces recensées par Lang à cette époque, une espece, $E$. cliffordae Chappuis 1932, présente une très courte épine à cet article. Ultérieurement, une deuxième espece, $E$ calypsonis Chappuis et Rouch 1959, fut décrite dont le mâle possède lui aussi une épine au baséoendopodite de la $P_{5}$. L'espèce découverte à Amiel constitue la troisième exception à une règle par ailleurs bien établie puisque le genre Elaphoidella sensu lato compte 150 especes environ. Avec un endopodite $P_{1}$ triarticulé, elle diffère d'E. cliffordae et $E$. calypsonis dont l'endopodite $P_{1}$ est biarticulé. Ainsi, le mâle d'Elaphoidella boui $\mathrm{n}$. sp. permet de distinguer aisément cette espèce parmi toutes les Elaphoidella.

La chaetotaxie des $P_{1}, P_{2}, P_{3}$ et de l'exopodite $P_{4}$ de la femelle d'E. boui, l'absence d'épine transformé au dernier article de l'exopodite $\mathrm{P}_{4}$ du mále sont autant de caractères qui incitent à placer cette espèce dans le groupe I (Lang 1948). La réduction de la chaetotaxie de l'endopodite $\mathrm{P}_{4}(0-111)$, qui paraît en contradiction avec la diagnose originelle de cé groupe, a déjà été signalée chez une espèce comme E. jojoi Petkovski 1982, rattachée au groupe I.

Si l'on adopte la classification proposée par Apostolov (1985), E. boti entre dans le groupe gracilis du genre Elaphoidella en raison des caractères suivants : endopodite $P_{1}$ triarticulé, endopodite $P_{4}$ biarticulé et présence de six soies et épines à l'article distal de l'exopodite $P_{4}$.

Au sein du groupe gracilis, l'espèce avec laquelle E. boui montre le plus d'analogie est Elaphoidella margaritae (Pesce et Apostolov, 1985), notamment 
en raison du fait qu'elles ont la même chaetotaxie particulière des $\mathbf{P}_{5}$ de la femelle, avec deux épines seulement à l'exopodite et quatre au baséondopodite. Toutefois, l'épine externe de ce baséoendopodite est plus longue que les trois épines internes chez $E$. bout alors que c'est l'inverse chez E. margaritae. Les femelles de ces deux especes ont en cormmun la chaetotaxie des exopodites $P_{1}, P_{2}, P_{3}, P_{4}$, de même que celle des endopodites $P_{1}$ et $P_{4}$ et du deuxième article de l'endopodite $\mathbf{P}_{2}$. A l'inverse d'E. bou, $E$. margaritae n'a pas d'épine interne au premier article des endopodites $P_{2}$ et $P_{3}$ et ne possède que cinq addendes au deuxième article de l'endopodite $P_{3}$ au lieu de six. Le mâle d'E. margaritae n'a que trois épines au deuxième article de l'endopodite $\mathbf{P}_{2}$ au lieu de quatre et ne possède pas d'épine au lobe interne de la $P_{5}$. Ces deux especes diffèrent enfin par l'ornementation des segments abdominaux et de l'oper. cule anal ainsi que par la forme des rames furcales.

Les autres espèces d'Elaphoidella présentant un exopodite de la $\mathbf{P}_{5}$ de la femelle armé de deux addendes seulement, appartiennent à des lignées très différentes et se distinguent donc aisément d'E. boui par au moins un caractère important dans le systeme proposé par Apostolov (op. cit.). E. romanica Kulhavy 1969 a un endopodite $\mathrm{P}_{1}$ biarticulé. $E$. infernalis Rouch 1970 ne possède pas d'endopodite $P_{4}$. E. pyrenaica Rouch 1970 a le dernier article de l'exopodite $\mathrm{P}_{4}$ avec cinq soies.

Les Elaphoidella dont l'exopodite $\mathbf{P}_{5}$ du mâle n'a que deux épines, comme $E$. winkleri (Chappuis 1928 a), E. javaensis (Chappuis 1928 b), E. turgisetosa Petkovski 1980 , ont une $P_{5}$ de la femelle avec trois épines à l'exopodite et n'appartiennent pas au groupe gracilis (sensu Apostolov). Quant au mâle décrit par Chappuis (1953 b) et qu'il rapporte à l'espèce pset. dophreatica, il est clair qu'il n'appartient pas à cette espèce (Kulhavy 1969, Petkovski 1972). Il se différencie du mâle d'E. boui par la présence d'une épine à l'angle distal interne du premier article de l'endopodite $\mathrm{P}_{4}$, par l'absence d'épine au basal de la $\mathrm{P}_{5}$ et par ses branches furcales très courtes.

E. boui se distingue facilement de l'autre espèce d'Elaphoidella (E. le ruthi meridionalis) qui vit dans la mème région par les caractères suivants : demier article de l'exopodite $P_{4}$ avec six addendes au lieu de cinq, deuxième article des endopodites $P_{2}$ et $P_{3}$ de la femelle avec une épine supplèmentaire, $P_{5}$ de la femelle avec 2 et 4 addendes au lieu de 3 et 3 .
Cette espèce est dédiée à Claude Bou, découvreur de la faune aquatique hypogée du sud-ouest du Massif Central.

\section{Travaux cités}

Apostolov (A.). 1985. - Elude sur quelques Copepodes Harpacticoïdes du genre Elaphoidella Chappuis, 1929 de Bulgarie avec une révision du genre. Acta Mus. Maced. Sc. Nat, 17 (7): 133-163.

Bou (C.) 1966. - Faune souterraine du sud-ouest du Massif Central. I. Contribution à la connaissance des Invertébrés caver. nicoles. Ann, Spéteoi., 21 (3) : 589-606.

Bou (C.). 1968. - Faune souterraine du sudouest du Massif Ceniral. II. Contribution a la connaissance de lo faune des eaux souterraines de l'Albigeois. Ann. Spéléol, 23 (2) : $441-473$.

Chappuis (P.A.). 1928 a. - Nouveaux Copépodes cavernicoles. Bull. Soc: Sc. Cluj, 4 (2) : 20-34.

Chappuis (P.A.) 1928 b. - Neue Harpacticiden aus Java. Treubia, $10(2-3): 271-283$.

Chappuis (P.A.). 1929. - Révision du genre Canlfocamptus. Bull. Soc. Sc. Cluj, 4 (2) : $41-50$.

Chappuis (P.A.). 1932 - Canthocamptinae nouveaux d'Afrique occidentale française. Bull. Soc. Sc. Cluj. $6: 413-420$.

Chappuis (P.A.). 1953 a. - Notes sur les Copepodes. 15. Un nouveau Parastenocaris de la vallé supérieure de la Weser (Hanovre). 16. Un nouvel Harpacticide cavernicole de Gréce. 17. Copépodes Harpacticoïdes de la grotte du Busc (Tarn-et-Garonne). Notes Biospeol., 8 : $81-90$.

Chappuis (P.A.). 1953 b. Nouveaux Crustacés troglobies de l'Italie du Nord. Mem. Mus. Civ. St. Nat. Verona, 4:1-12.

Chappuis (P.A.) et Rouch (R.). 1959. - Deux nouveaux Copépodes cavernicoles des Pyrénées. Ann. Spéléol., 14 (1-2) : 213-218.

Kulhavy (V.). 1969. - Uber Höhlenharpacticiden aus dem Rumänischen Banat. Acta Soc. Zool. Bohemoslov'. 33 (1) : 5-14.

Lang (K.). 1948. - Monographie der Harpacticiden, I, II : 1682 p. (Häkan Ohisson, Lund).

Lebreton (B.) \& Dussart (B.). 1984. -- Faune souterraine du département de la Dordogne. I. Crustaces Copépodes. Mém. Biospéol., $11: 275-280$.

Pesce (G.L.) \& Apostolov (A.). 1985. - Elaphoidello margarirae sp. n., a new phreatobitic harpacticoid from subterranean waters of Thailand (Crustacea, Copepoda, Canthocamptidae). Acta 7ool. Bulgarica, $28: 70-75$.

Petkovski (T.K.). 1972. - Zur Copepodenfauna der Höhlen von Banat. Acta Mizi virued. Sc. Nat., 13 (2): 21-38.

Petko'ski (T.K.). 1980. - Fünf neue Elaphoidella - Arten (Copepoda Harpacticoida) aus den subterranen Gewässern von Kuba. Acta Mus. Maced. Sc. Nat., $16(2): 33-70$.

Petkovski (T.K.). 1982. - Weitere neue Elaphoidella - Arten (Copepoda Harpacticoida) aus den subterranen Binnengewässern von Kuba. Acta Mis. Maced. Sc. Nal., 16 (5) : 139-174.

Rouch (R.). 1968. - Contribution à la connaissance des Harpacticides hypogés (Crustacés, Copépodes). Ann. Spéléot., 23 (1): 5-167.

Rouch (R.). 1970. - Recherches sur les eaux souterraines. 8. Harpacticides nouveaux des eaux souterraines des Pyrénées. Ann. Spéléol., 25 (1): 139.154.

Rouch (R.). 1986. - Copepoda : les Harpacticoides souferrains des eaut douces continentales. In : L. Botosaneanu ed., Stygofauna mundi : 321.355 (E. J. Brill, Leiden). 